\title{
Age at Death and Causes of Death in Patients with Huntington Disease in Norway in 1986-2015
}

\author{
Olga Karin Solberga ${ }^{\mathrm{a}}$, Petra Filkukováa ${ }^{\mathrm{a}}$ Jan C. Frich ${ }^{\mathrm{b}, \mathrm{c}}$ and Kristin J. Billaud Feragen ${ }^{\mathrm{a}, *}$ \\ ${ }^{a}$ Centre for Rare Disorders, Oslo University Hospital, Oslo, Norway \\ ${ }^{\mathrm{b}}$ Department of Neurology, Oslo University Hospital, Oslo, Norway \\ ${ }^{\mathrm{c}}$ Institute of Health and Society, University of Oslo, Oslo, Norway
}

\begin{abstract}
.
Background: The literature offers discrepant findings regarding age at death in individuals with Huntington disease (HD). Objective: To study the age at death and causes of death in males and females with a diagnosis of HD in Norway.

Methods: Registry study of deaths in 1986-2015 using data from two national registries: the Norwegian Cause of Death Registry (NCDR) and the registry of the Centre for Rare Disorders (CRD), Oslo University Hospital.

Results: Mean age at death for individuals with HD was found to be 63.9 years (NCDR) and 61.7 years (CRD), compared to a mean of 76.9 years in the general population (NCDR). There were no significant gender differences for age at death in individuals with HD. The significant increase in age at death within the general population from 1986 to 2015 was not observed in individuals with HD. In 73.5\% of individuals with HD, the underlying cause of death was HD, followed by cardiovascular diseases, cancer and respiratory diseases. The most common immediate cause of death was respiratory diseases $(44.2 \%)$. Suicide was a more common cause of death in the population with HD (2.3\%) compared to the general population (1.3\%).

Conclusion: The age at death of individuals with HD was stable over a period of 30 years and 13.3 years lower than in the general population. Longer life expectancy for females from the general population was not found in females with HD. Suicide was more common among individuals with HD compared to the general population.
\end{abstract}

Keywords: Age at death, causes of death, Huntington disease, suicide

\section{INTRODUCTION}

Huntington disease (HD) is an autosomal dominant neurological disease caused by an expanded CAG repeat in the Huntingtin gene [1]. The disease is characterized by progressive functional decline and motor, psychiatric and cognitive symptoms [2,3]. Clinical symptoms of HD usually develop between

\footnotetext{
${ }^{*}$ Correspondence to: Dr. Kristin J. Billaud Feragen, Centre for Rare Disorders, Oslo University Hospital, P.O. Box 4950 Nydalen, 0424 Oslo, Norway. Tel.: +47 230753 42; E-mail: krifer@oushf.no.
}

30 and 50 years of age and disease duration is approximately 15-20 years from first clinical symptoms to complete care dependency and death.

Clinical experience indicates that questions related to prognosis and life expectancy are central for affected individuals and their families. Population studies based on national registries may provide important data for the investigation of rare conditions, and have been used in several studies on HD. Based on registry data, two studies found that the mean age at death was 56 years in a population of individuals with HD from Southern Italy [4] and in the United 
States [5]. A study of age at death in Serbia found a slightly lower mean age at death of 51.0 years, and a gender difference of five years, with lower mean age at death of 49.1 in males [6].

Other studies have investigated immediate and underlying causes of death in patients with HD [7-11]. In three studies [5, 7, 11], the most common immediate cause of death was pneumonia, followed by cardiovascular diseases. Suicide has also been reported to be more prevalent in the HD population compared to the general population [7-9], as already described by Huntington in 1872 [12]. An early Norwegian study reported a suicide rate of $2.2 \%$ between 1916 and 1975 in a population of individuals with HD, based on data from a national registry [9]. A Danish study, also based on data from national death certificates, revealed that in cases of HD as an underlying cause of death, $5.6 \%$ had committed suicide, compared to $2.7 \%$ in the general population [7]. Knowledge about causes of death in individuals with HD could inform health care services about medical risk and treatment needs in patients with $\mathrm{HD}$, and in cases of treatable or preventative conditions, provide substantial benefits to the patients [10]. Knowledge about age at death and causes of death is also relevant in at health policy levels, to increase awareness of the impact of HD [13].

The aim of the present study was therefore to study the age at death and cause of death in males and females with a diagnosis of HD in Norway in the period 1986-2015. The following research questions were investigated: How did the number of cases of death and age at death among individuals with HD develop over 30 years? What were the frequencies of direct and underlying causes of death in individuals who have a diagnosis of HD on their death certificate? Developmental trends in the number of deaths and age at death among the HD population are compared with the general Norwegian population in the same period.

\section{METHODS}

The present study was based on data from two national registries that provided information about age at death of individuals with HD in the period 1986-2015: the Norwegian Cause of Death Registry (NCDR) at the Norwegian Institute of Public Health and the patient registry at the Centre for Rare Disorders (CRD), Oslo University Hospital, Norway. The 30-year period from 1986 to 2015 was chosen because we expected a higher diagnostic quality and completeness of data from the mid-1980 s.

\section{Description of data}

The Norwegian Cause of Death Registry (NCDR) provides information on date and cause of death (immediate and underlying) in the Norwegian population. Information about causes of death in individuals with HD could be extracted from the registry. Underlying cause of death is defined as "the disease or injury that initiated the chain of events leading directly to death, or the external circumstances of the accident or violence that was the cause of the fatal injury". Immediate cause of death is defined as "the disease, injury or condition directly leading to death and which was caused by the underlying cause of death" (WHO, International classification of diseases). As an example, difficulties with swallowing due to HD (underlying cause) may lead to aspiration pneumonia and sepsis (immediate cause of death).

Anonymous data were obtained from the NCDR regarding age at death and immediate and underlying cause of death in individuals with HD in the period 1986-2015. Individuals with HD were identified in the NCDR when HD was listed on the death certificate as one of the diagnoses. A total of 559 individuals with HD were identified through the NCDR in this period. Data about age at death and causes of death in the general Norwegian population were obtained for the same period 1986-2015.

The Centre for Rare Disorders (CRD) is a multidisciplinary, nationwide competence center offering information, counselling and courses for nearly 70 rare disorders, HD being one of these. The center's services are aimed at individuals with a rare disorder and their families, as well as professionals working with these disorders. Individuals and their families may contact the center without referral. Registration is voluntary, and the registry therefore contains only information about those who have contacted the center for counselling or in search of information, and who also have consented in being included in the registry. Among all individuals registered with HD, a total of 439 cases of death were identified in the period 1986-2015.

The NCDR and the CRD are both operating at a national level. Therefore, most patients with HD are expected to be found in both registries. Analyses were therefore run separately for both registries, in order to avoid bias. 


\section{Procedure}

Data on gender, age at death and year of death were registered in the CRD's patient registry. Causes of death are not systematically registered at the CRD. Results (gender, age at death, number of deaths per year) were compared to figures from the NCDR during the same period (1986-2015), based on anonymous data.

\section{Statistical analyses}

The statistical analyses were performed using SPSS Statistics version 23 (IBM Corp, Armonk, NY). Descriptive statistics were calculated. Chi-square tests were used to calculate differences between dichotomous groups, while independent sample $t$-tests were used when calculating differences between continuous variables.

\section{RESULTS}

\section{Age at death}

In the period 1986-2015 we identified a total of 559 deceased individuals with HD in the NCDR and 439 deceased individuals in the HD registry of the CRD (Table 1). According to the data from NCDR, as many as $14.6 \%(n=82)$ of the individuals with HD reached the age of 80 years or higher, of which $2.3 \%$ $(n=13)$ reached an age above 90 years. We also found that $5 \%(n=28)$ of individuals with HD died before the age of 40 .

As can be seen in Table 2, the mean age at death of individuals with HD in the period 1986-2015 in the NCDR was 63.9 years, compared to a mean age at death of 76.9 years in the general population $(t(1291214)=19450, p<0.0001)$.

When comparing the two registries we found that individuals with $\mathrm{HD}$ registered at the CRD lived about two years shorter than the ones in the NCDR. The largest discrepancy between the two registries was found for a small group of individuals who died of HD before reaching the age of 30 (80\% of the cases in this age group registered in the NCDR was missing in the CRD's registry), followed by a larger group of individuals who died in their $80 \mathrm{~s}(55.1 \%$ missing in the CRD's registry) and $90 \mathrm{~s}$ (46.2\% missing).

Data from the NCDR showed (Table 2) that there was no significant difference in the age at death between males and females with HD $(\mathrm{NCDR}: t(557)=1.68 ; p=0.094 ; \mathrm{CRD} t(437)=0.62$, $p=0.537)$. In contrast, females in the general population lived significantly longer than males $(t(1290655)=231.33, p<0.001)$. Males with HD died on average at the age of 62.9 , compared to 73.7

Table 1

Individuals with HD deceased in 1986-2015: Comparison of data from the Norwegian Cause of Death Registry (NCDR) and the registry of the Centre for Rare Disorders (CRD)

\begin{tabular}{|c|c|c|c|c|c|c|c|}
\hline \multirow[t]{2}{*}{ Age } & \multicolumn{3}{|c|}{ NCDR } & \multicolumn{3}{|c|}{ CRD } & \multirow{2}{*}{$\begin{array}{c}\text { Difference } \\
\text { (NCDR-CRD) } \\
\mathrm{N} \\
\end{array}$} \\
\hline & $\begin{array}{l}\text { Males } \\
\mathrm{N}(\%)\end{array}$ & $\begin{array}{l}\text { Females } \\
\mathrm{N}(\%)\end{array}$ & $\begin{array}{c}\text { Total } \\
\mathrm{N}(\%)\end{array}$ & $\begin{array}{l}\text { Males } \\
\mathrm{N}(\%)\end{array}$ & $\begin{array}{r}\text { Females } \\
\mathrm{N}(\%)\end{array}$ & $\begin{array}{l}\text { Total } \\
\text { N (\%) }\end{array}$ & \\
\hline 10-19 & $1(0.4)$ & $0(0)$ & $1(0.2)$ & $0(0)$ & $0(0)$ & $0(0)$ & 1 \\
\hline $20-29$ & $4(1.5)$ & $0(0)$ & $4(0.7)$ & $1(0.5)$ & $0(0)$ & $1(0.2)$ & 3 \\
\hline $30-39$ & $9(3.3)$ & $14(4.9)$ & $23(4.1)$ & $5(2.3)$ & $9(4.1)$ & $14(3.2)$ & 9 \\
\hline $40-49$ & $40(14.7)$ & $29(10.1)$ & $69(12.3)$ & $37(17)$ & $27(12.2)$ & $64(14.6)$ & 5 \\
\hline $50-59$ & 57 (20.9) & $70(24.5)$ & $127(22.7)$ & $56(25.7)$ & $63(28.5)$ & $119(27.1)$ & 8 \\
\hline $60-69$ & $74(27.1)$ & 59 (20.6) & $133(23.8)$ & $61(28)$ & $55(24.9)$ & $116(26.4)$ & 17 \\
\hline $70-79$ & $53(19.4)$ & $67(23.4)$ & $120(21.5)$ & $43(19.7)$ & $44(19.9)$ & $87(19.8)$ & 33 \\
\hline $80-89$ & $30(11)$ & 39 (13.6) & $69(12.3)$ & $11(5)$ & $20(9)$ & $31(7.1)$ & 38 \\
\hline 90-99 & $5(1.8)$ & $8(2.8)$ & $13(2.3)$ & $4(1.8)$ & $3(1.4)$ & $7(1.6)$ & 6 \\
\hline Total & $273(100)$ & $286(100)$ & $599(100)$ & $218(100)$ & $221(100)$ & $439(100)$ & 120 \\
\hline
\end{tabular}

Table 2

Mean age at death in Norway: Comparison of individuals with HD and the Norwegian general population

\begin{tabular}{lccc}
\hline & Males $M(S D)$ & Females $M(S D)$ & Total $M(S D)$ \\
$\left(N_{N C D R}=273 ;\right.$ & $N_{N C D R}=559 ;$ & $N_{C R D}=439 ;$ \\
& $N_{C R D}=218 ;$ & $N_{C R D}=221 ;$ & $63.9(14.2)$ \\
& $\left.N_{\text {population }}=642734\right)$ & $\left.N_{\text {population }}=647923\right)$ & $N_{\text {population }}=1290657$ \\
\hline Individuals with HD 1986-2015 (NCDR) & $62.9(13.9)$ & $64.9(14.3)$ & $61.7(12.9)$ \\
Individuals with HD 1986-2015 (CRD) & $61.3(12.5)$ & $62.1(13.3)$ & $76.9(15.8)$ \\
Population 1986-2015 (NCDR) & $73.7(16.3)$ & $80.0(14.6)$ & \\
\hline
\end{tabular}


years in the general population $(t(643005)=10.95$, $p<0.001)$. Females with HD had a mean age at death of 64.9 years, compared to 80.0 years in the general population $(t(648207)=17.49, p<0.001)$. On average, males and females with HD died 10.8 and 15.1 years earlier, respectively, compared with males and females in the general Norwegian population. Figures from the NCDR and the CRD's registry indicate a similar gender-wise proportion of deceased males and females with HD (NCDR: $48.8 \%$ males, $51.2 \%$ females; CRD: $49.7 \%$ males, $50.3 \%$ females), which corresponds to the ratio of deceased males and females in the general population during the same time period $(49.8 \%$ males, $50.2 \%$ females; NCDR: $\chi^{2}(1, \mathrm{~N}=559)=0.207, p=0.649 ; \mathrm{CRD}$ : $\left.\chi^{2}(1, \mathrm{~N}=439)=0.004, p=0.953\right)$.

Due to a relatively low number of deceased individuals with HD per year, mean age at death was calculated in five-year intervals (Table 3, Fig. 1). In order to investigate whether the age at death significantly changed between 1986 and 2015, we compared the mean age at death in the first five-year interval (1986-1990) with the mean age at death in the last five-year interval under study (2011-2015). For individuals with HD, the mean age at death in 1986-1990 $(M=62.8, S D=13.5)$ was not significantly different from the mean age at death of individuals with HD in 2011-2015 $(M=64.4, S D=14.7 ; t(191)=0.75$, $p=0.455$ ). This finding was consistent also for males and females separately, as we found no statically significant differences between the first and the last time period (Males: $t(98)=0.26, p=0.798$; Females: $t(91)=1.13, p=0.262)$.

In the general population, there was a statistically significant difference of four years in the mean age at death between the two time periods of 1986-1990 and 2011-2015 $(t(427705)=92.48$, $p<0.001)$. This difference was significant for both males $(t(216224)=59.41, p<0.001)$ and females $(t(211479)=65.64, p<0.001)$. In other words, the significant increase in age at death in the general population was not found for individuals with HD. During the period of 1986-1990, the disparity in age of death between individuals with HD and the general population was 11.6 years, whereas this difference was 14.5 years in the period 2011-2015. In both groups (individuals with HD and the general population), the dispersion of the values around the mean (standard deviation) was similar, with slightly more variability in age at death in the general population $\left(\mathrm{SD}_{\mathrm{HD}}=14.2\right.$ vs $\left.\mathrm{SD}_{\text {Genpop }}=15.8\right)$.

During the period of 1986-2015, we observed an increasing number of deceased individuals with HD, from 70 in 1986-1990 to 123 in 2011-2015 (Fig. 2).

An increased number of diseased individuals with HD could not be explained by an increasing number of deceased individuals in general, as the number of

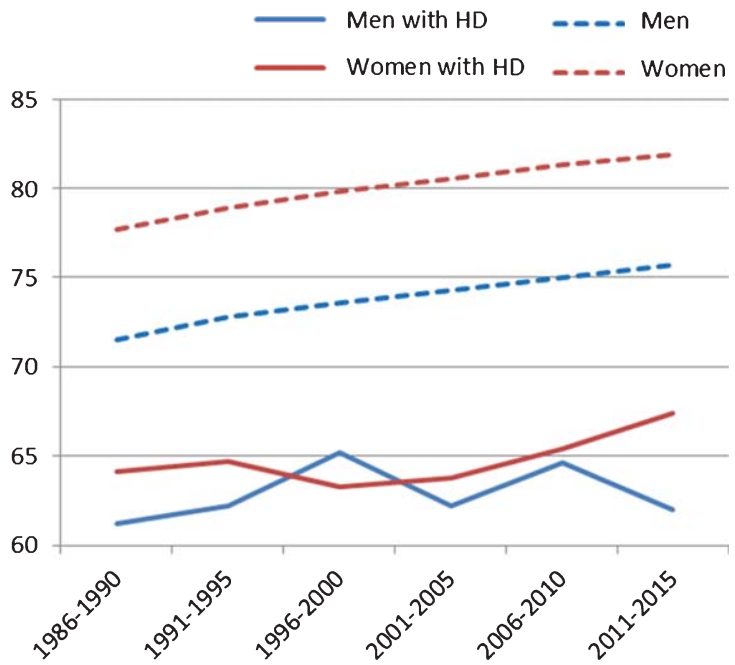

Fig. 1. Development of age at death in individuals with HD and general population (1986-2015). Source: NCDR.

Table 3

Age at death for five-year intervals: Comparison between individuals with $\mathrm{HD}(n=559)$ and the general population $(n=1.290 .657)$ in $1986-2015$

\begin{tabular}{|c|c|c|c|c|c|c|}
\hline & \multicolumn{6}{|c|}{ Age at death } \\
\hline & \multicolumn{2}{|c|}{$\begin{array}{c}\text { Males } \\
M_{\text {age }}(S D)\end{array}$} & \multicolumn{2}{|c|}{$\begin{array}{c}\text { Females } \\
M_{\text {age }}(S D)\end{array}$} & \multicolumn{2}{|c|}{$\begin{array}{c}\text { Total } \\
M_{\text {age }}(S D)\end{array}$} \\
\hline & $\mathrm{HD}$ & Population & $\mathrm{HD}$ & Population & HD & Population \\
\hline 1986-1990 & $61.2(13.2)$ & $71.5(16.8)$ & $64.1(13.8)$ & $77.7(15.2)$ & $62.8(13.5)$ & $74.4(16.4)$ \\
\hline 1991-1995 & $62.2(14.0)$ & $72.8(16.2)$ & $64.7(14.5)$ & $78.9(14.6)$ & $63.6(14.3)$ & $75.8(15.7)$ \\
\hline 1996-2000 & $65.2(14.3)$ & $73.6(16.2)$ & $63.3(13.7)$ & $79.8(14.4)$ & $64.2(13.9)$ & 76.7 (15.6) \\
\hline 2001-2005 & $62.2(13.8)$ & $74.3(16.3)$ & $63.8(14.7)$ & $80.5(14.4)$ & $63.1(14.2)$ & 77.5 (15.6) \\
\hline 2006-2010 & $64.6(13.0)$ & $75.0(16.1)$ & $65.4(15.3)$ & $81.3(14.3)$ & $65.0(14.1)$ & $78.3(15.5)$ \\
\hline 2011-2015 & $62.0(14.9)$ & $75.7(15.8)$ & $67.4(14.0)$ & $81.9(14.2)$ & $64.4(14.7)$ & $78.9(15.3)$ \\
\hline
\end{tabular}

Source: NCDR. 


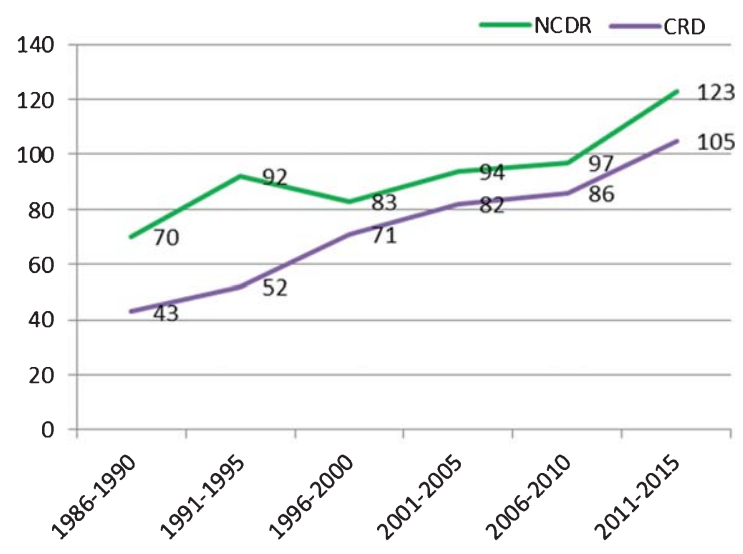

Fig. 2. Number of cases of death of individuals with HD in 1986-2015: Comparison of two registries.

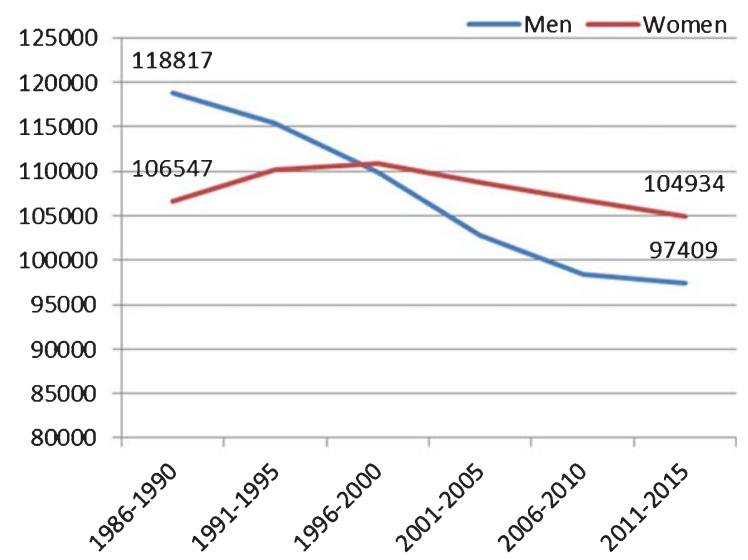

Fig. 3. Number of cases of death in Norwegian population in 1986-2015. Source: NCDR. Population size in Norway: 1986:4159187, 2015:5165802 [20].

deceased individuals in the Norwegian population has been steadily decreasing, as can be seen in Fig. 3 (1986-1990: $n=225364$; 2011-2015: $n=202343$ ).

\section{Differences between the two registries}

Figure 2 illustrates that a higher number of individuals with HD were registered at the NCDR compared to the CRD in the given time period. The difference between the two databases has decreased over time.

\section{Cause of death}

When examining information from the NCDR, HD was registered as the underlying cause of death in $73.5 \%(n=411)$ individuals with HD (Table 4). For the remaining $26.5 \%(n=148)$, the subsequent causes of death were cardiovascular diseases $(10.2 \%$; $n=57)$, followed by cancer $(4.3 \% ; n=24)$ and respiratory diseases $(3.8 \% ; n=21)$. A small proportion of individuals with HD died from injuries or poisoning $(3.2 \%, n=18)$, whereas in $2.3 \%(n=13)$, suicide was registered as an underlying cause of death. Suicide was mentioned more often in males with $\mathrm{HD}(3.7 \%$; $n=10)$ than in females $(1.0 \% ; n=3)$.

In the general Norwegian population (1986-2015), the order of underlying causes of death was similar to the one found in the HD group: cardiovascular diseases $(40.3 \%)$, cancer $(24.2 \%)$ and respiratory diseases $(9.8 \%)$. Injuries and poisoning was reported for $4.4 \%$, whereas suicide constituted $1.3 \%$ of causes of death in the general population. Males died by suicide more frequently $(1.9 \%)$ than females $(0.7 \%)$.

The incidence of suicides was significantly higher in individuals with HD compared with the general population; $\chi^{2}(1, N=559)=4.34, p=0.037$. When investigating males and females separately, the difference in suicide prevalence was significant only for males $\left(\chi^{2}(1, N=273)=4.40, p=0.036\right)$, but not for females $\left(\chi^{2}(1, N=286)=0.42, p=0.518\right)$.

Individuals with HD had a lower mean age at death than the general population for all underlying causes of death except for suicide, where a higher mean age was found in individuals with $\mathrm{HD}\left(\mathrm{M}_{\mathrm{HD}}=55.0\right.$, $\mathrm{SD}=8.6 ; \mathrm{M}_{\text {Genpop }}=46.0, \mathrm{SD}=18.5$ ). A lower mean age at death was also the case for individuals with HD dying of "other injuries and poisoning" (Table 4), first of all in females. However, these differences were not statistically significant (Total sample: $t(57391)=1.82, p=0.069$; Males: $t(32758)=0.77$, $p=0.441$; Females: $t(24631)=1.83, p=0.068)$.

As can be seen in Table 5, the most common immediate cause of death among individuals with HD was respiratory diseases $(44.2 \%)$, followed by HD $(21.5 \%)$.

\section{DISCUSSION}

The present study examined the age at death in individuals diagnosed with HD, using data from two national registries. Whereas the NCDR includes all cases of death in Norway, by age, gender, and cause of death, the CRD registry is voluntary, lacks information of cause of death, and contains only information about individuals who had been in contact with the center. The deceased individuals with HD registered at the CRD lived about two years shorter than individuals with HD registered at the NCDR, which may suggest that those who were diagnosed with $\mathrm{HD}$ at a 


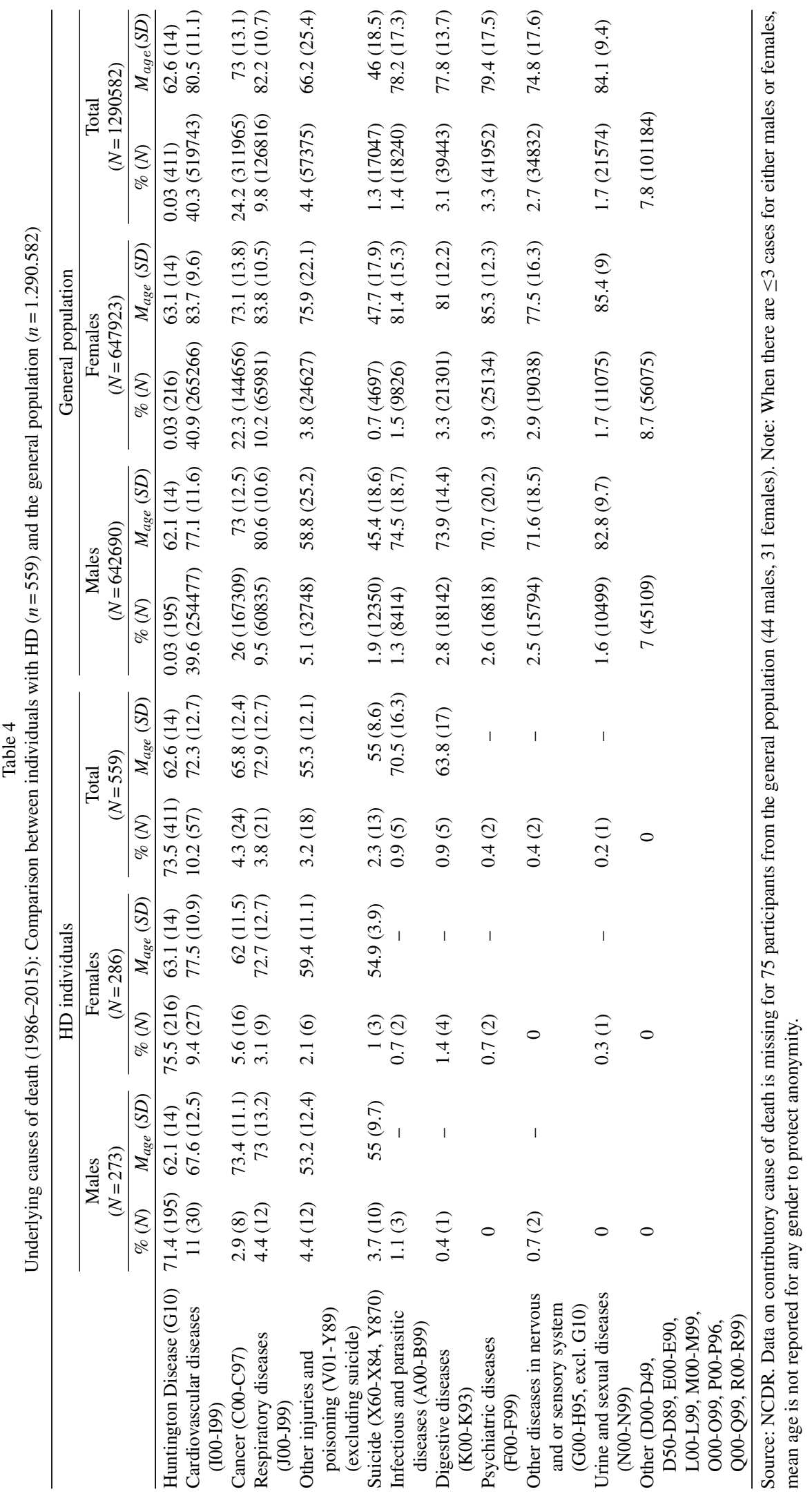


Table 5

Immediate cause of death: individuals with HD (NCDR)

\begin{tabular}{|c|c|c|c|c|c|c|}
\hline & \multicolumn{2}{|c|}{$\begin{array}{l}\text { Males with HD } \\
\quad(N=123)\end{array}$} & \multicolumn{2}{|c|}{$\begin{array}{l}\text { Females with HD } \\
\quad(N=110)\end{array}$} & \multicolumn{2}{|c|}{$\begin{array}{c}\text { Total } \\
(N=233)\end{array}$} \\
\hline & $\%(N)^{*}$ & $M_{\text {age }}(S D)$ & $\%(N)^{*}$ & $M_{\text {age }}(S D)$ & $\%(N)^{*}$ & $M_{\text {age }}(S D)$ \\
\hline Respiratory diseases (J0-J99) & $52.8(65)$ & $64.5(14.3)$ & $34.5(38)$ & $65.2(15.9)$ & $44.2(103)$ & $64.8(14.8)$ \\
\hline Huntington Disease (G10) & $12.2(15)$ & $62.1(14.6)$ & $31.8(35)$ & $64.7(13.6)$ & $21.5(50)$ & $63.9(13.8)$ \\
\hline $\begin{array}{l}\text { Symptoms, signs and abnormal clinical and laboratory } \\
\text { findings, not elsewhere classified (R00-R99) }\end{array}$ & $7.3(9)$ & $62.9(7.5)$ & $14.5(16)$ & $64.6(12.5)$ & $10.7(25)$ & $64(10.8)$ \\
\hline Cardiovascular diseases (I00-I99) & $12.2(15)$ & $69.6(11.9)$ & $7.3(8)$ & $78.8(11.7)$ & $9.9(23)$ & $72.8(12.4)$ \\
\hline $\begin{array}{l}\text { Injury, poisoning and certain other consequences of } \\
\text { external causes (S00-T98) }\end{array}$ & $7.3(9)$ & - & $2.7(3)$ & & $5.2(12)$ & $54.6(12.1)$ \\
\hline Infectious and parasitic diseases (A00-B99) & $3(4)$ & $60.9(7.1)$ & $5.5(6)$ & $71(16.2)$ & $4.3(10)$ & $66.9(13.8)$ \\
\hline Endocrine, nutritional and metabolic diseases (E00-E90) & $2.4(3)$ & & $3.6(4)$ & & $3.0(7)$ & $61.6(14.3)$ \\
\hline Suicide $(\mathrm{X} 60-\mathrm{X} 84, \mathrm{Y} 87)$ & $4.1(5)$ & & $1.8(2)$ & & $3.0(7)$ & $56.3(9.9)$ \\
\hline $\begin{array}{l}\text { Other diseases in nervous and or sensory system } \\
\text { (G00-H95, excl. G10) }\end{array}$ & $2.4(3)$ & & & & $1.3(3)$ & \\
\hline Digestive diseases (K00-K93) & $0.8(1)$ & & $1.8(2)$ & & $1.3(3)$ & \\
\hline $\begin{array}{l}\text { Other injuries and poisoning (V01-Y89) (excluding } \\
\text { suicide) }\end{array}$ & $1.6(2)$ & & $0.9(1)$ & & $1.3(3)$ & \\
\hline Cancer (C00-C97) & $0.8(1)$ & & $0.9(1)$ & & $0.9(2)$ & \\
\hline
\end{tabular}

* Some individuals had more than one immediate cause of death listed in their death certificate, hence leading to a sum exceeding $100 \%$. In all cases when suicide was mentioned as immediate cause of death in individuals with HD, it was also listed as underlying cause of death (NCDR). Data on immediate cause of death is missing for 150 males (54.9\% of the sample), 176 females (59.9\% of the sample). Note: When there are $\leq 3$ cases for either males or females, mean age is not reported for any gender.

higher age were less likely to seek contact with the CRD. Otherwise, there were no differences between the two registries: both showed no increase in age at death over years, no difference in age at death between males and females, and an increasing number of deceased individuals with HD. Differences between the two registries suggests that almost $80 \%$ of those diagnosed with HD in Norway have been in contact with the CRD for counseling and followup. The largest gaps between the two registries were found for those dying of HD before the age of 30 and after the age of 80 years.

Our results show that the number of deaths of individuals with a diagnosis of HD increased during the period under study. Several factors may explain this finding. First, neurological diseases such as HD may be diagnosed more frequently and more precisely than a couple of decades ago. Secondly, death certificates may have become more exhaustive over time, and physicians who fill in the certificates may also have easier access to central medical information about the patients. Additionally, life expectancy increased in the Norwegian population during this period, which could also potentially lead to an increased number of individuals with late onset HD [14]. Still, if the increase in the number of cases was due to individuals with late manifest HD, one would expect that the mean age at death for individuals with HD would increase over the decades, which was not observed in the present study.
Despite a population growth, the number of deceased individuals in Norway had decreased from 1986 to 2015. A decrease in the number of deaths in the general population may be explained by better health conditions and health care, an effect that did not seem beneficial for individuals with HD: while life expectancy rose with four years in the general population in the given 30-year period, no significant difference in mean age at death was found in the group with HD during the same period. Consequently, an increasing disparity in mean age at death from HD and age at death in the general population is evident. During the period of 1986-1990, the gap in age at death between individuals with HD and the general population was 11.6 years, whereas this gap had increased to 14.5 years in 2011-2015. Further, there was no difference in age at death between males and females with HD, as has been reported previously [5], which means that the relative loss of years is larger for females with $\mathrm{HD}$, as they live longer than males in the general population. In other words, improved health conditions and health care leading to an extended life expectancy in the general population, does not seem to benefit patients with HD, as was also observed in a Dutch study in 1999 [15]. Nevertheless, improved care may reduce suffering associated with some of the disease's symptoms, and could therefore have the potential to decrease caregiver burden and increase quality of life in patients with HD [10]. Future research is however needed to confirm this. 
It should be noted that individuals with HD who died during the given time period belonged to younger birth cohorts (later years of birth) than the general population who died during the same time period. If data had been available so that same birth cohorts could be compared, the relative loss of years for an individual with HD would probably have been even larger.

For individuals registered with HD on their death certificate, HD was the most common underlying cause of death $(73.5 \%)$, and respiratory diseases were the most common immediate cause of death $(64.5 \%)$. In two other studies $[4,11]$, pneumonia and diseases of the respiratory system were also reported as the main primary cause of death in individuals with HD (42\% and 55\% respectively), followed by cardiovascular complications ( $26 \%$ and $10.9 \%$ ). However, as in the present study, the primary cause of death was unspecified in many cases, implying uncertainties associated with these findings. Differences between studies regarding causes of death may be explained by different levels of care and diagnostic sensitivity across countries and studies. Countries can also be expected to differ in terms of how causes of death are registered, and diagnostic terms may also differ across registries, leading to a variation in the frequency of reported causes of death.

Our study found that the three subsequent underlying causes of death were equal to the most common underlying causes of death in the general population: cardiovascular diseases, cancer, and respiratory disease. However, even if the order of causes of death were similar, numbers differed. This could probably be explained by a lower mean age at death in patients with HD, which means that individuals who could be at risk of developing cancer or cardiovascular diseases may die from HD before developing other medical conditions.

Individuals with HD had a lower mean age at death than the general population for all underlying causes of death except for suicide. In the literature, suicide figures in patients with HD are found to vary across studies, ranging from $0.6 \%$ to $10 \%$ [16]. In the present study, suicide was more prevalent among males with HD $(3.7 \%)$ than among males from the general population $(1.9 \%)$, whereas there was no difference in the prevalence of suicide between females with $(1 \%)$ and without HD $(0.7 \%)$. Suicide was almost four times more frequent underlying cause of death for males compared with females with HD, while in the general population, suicide was almost three times more frequent in males than in females. Another interesting finding was that individuals with $\mathrm{HD}$ committed suicide at a higher age (7-10 years later) than the general population, with a mean age of 55.0 years for males and 54.9 years for females. This finding may suggest that those at risk for suicide within the HD group differ from the general population in terms of psychological risk, probably explained by concerns related to the development of the disease and experiences of function decline $[17,18]$. On the other hand, individuals who feared that they might have inherited HD could also have committed suicide in their thirties or forties, before having been diagnosed. This group would not be registered with HD when committing suicide, leading to a registered increase in the mean age of death by suicide for individuals with $\mathrm{HD}$ and lower suicide figures than what is really the case. Another interesting subgroup that could potentially be associated with suicide figures, are those who were registered with "other injuries and poisoning" as underlying cause of death. On the other hand, the literature has demonstrated that this group may also include patients that have died as a result of falls [19]. Individuals with HD dying of other injuries and poisoning had a mean age at death that was lower than in the general population, most notably for females (by 16.5 years). This difference was however not statistically significant, probably due to small HD subsamples.

Future research should aim at having more complete information of causes of death, including immediate and contributory causes of death. It would also be interesting to have information on the CAG repeat length and investigate its relationship to the age of death. Other factors that could be of potential interest for future research is information about potentially protective factors of the patient's environment, such as marital status which has been found to be associated with later onsets of HD [9]. Another area of future research could be to explore not only the age at death, but also disease duration and factors associated with slower progress of the disease.

\section{Strengths and limitations}

The main strength of the present study was its access to relatively complete data from national registries containing information about individuals diagnosed with HD. Further, comparing information from two different registries provided a quality check of CRD's voluntary patient registry. Since patients could be listed in both registries, analyses were run separately for data from the NCDR and the CRD, 
hence no bias due to a potential double registration was present. Still, some limitations need to be acknowledged. First, and as expected, the number of registered individuals with $\mathrm{HD}$ at the CRD was lower than the national death registry (NCDR). A lower number of individuals with HD in the CRD registry could indicate that some individuals and their families do not have information about their possibility to contact the CRD, or they feel they do not need counselling and information from the center. Another explanation could be that families wish to hide the disease that runs in the family, a medical condition that still generates shame, and therefore do not contact the center. Further, questions could be asked regarding whether HD can be expected to be registered correctly when cause of death is not directly linked to the disease and hence is not registered on the death certificate. As an example, suicide in individuals who fear to have inherited the disease, but have not yet been diagnosed, would be registered within the general population. Last, the data from the registries did not contain any individual information that could potentially have helped interpret results, such as for example CAG repeat data or time of diagnosis.

\section{Conclusion}

The age at death of individuals with HD was stable over a period of 30 years, which implies that the disparity between age at death from HD and age at death in the general population was found to be increasing. No gender difference in age at death was observed in patients with $\mathrm{HD}$, in contrast to what is found in the general population, which indicates that females with HD have a larger average loss of years than males. Suicide was more common among individuals with HD compared to the general population, particularly in males. Knowledge about a reduced life expectancy for individuals with HD compared with the general population illustrates the impact of HD on those affected.

\section{CONFLICT OF INTEREST}

The authors have no conflict of interest to report.

\section{COMPLIANCE WITH GUIDELINES}

No human or animal experimentation were necessary for the present work.

\section{REFERENCES}

[1] Bates G, Tabrizi S, Jones L. Huntington's disease. 4th ed. Oxford: Oxford University Press, 2014.

[2] Harrington DL, Rubinov M, Durgerian S, Mourany L, Reece C, Koenig K, Bullmore E, Long JD, Paulsen JS for the PREDICT-HD investigators of the Huntington Study Group and Rao SM. Network topology and functional connectivity disturbances precede the onset of Huntington's disease. Brain. 2015;138:2332-46.

[3] Eddy CM, Parkinson EG, Rickards HE. Changes in mental state and behaviour in Huntington's disease. Lancet Psychiatry. 2016;3(11):1079-86.

[4] Rinaldi C, Salvatore E, Giordano I, De Matteis S, Tucci T, Cinzia VR, Rossi F, Castaldo I, Morra VB, Di Maio L, Filla A, De Michele G. Predictors of survival in a Huntington's disease population from southern Italy. Can J Neurol Sci. 2012;39(1):48-51.

[5] Lanska DJ, Lavine L, Lanska MJ, Schoenberg BS. Huntington's disease mortality in the United States. Neurology. 1988;38(5):769-72.

[6] Pekmezovic T, Svetel M, Maric J, Dujmovic-Basuroski I, Dragasevic N, Keckarevic M, Romac S, Kostic VS. Survival of Huntington's disease patients in Serbia: Longer survival in female patients. Eur J Epidemiol. 2007;22(8):523-6.

[7] Sørensen AS, Fenger K. Causes of death in patients with Huntington's disease and in unaffected first degree relatives. J Med Genet. 1992;29(12):911-4.

[8] Hubers AA, van Duijn E, Roos RA, Craufurd D, Rickards $\mathrm{H}$, Bernhard Landwehrmeyer $\mathrm{G}$, van der Mast RC, Giltay EJ; REGISTRY investigators of the European Huntington's Disease Network. Suicidal ideation in a European Huntington's disease population. J Affect Disord. 2013;151(1):248-58.

[9] Saugstad L, Ødegård Ø. Huntington's chorea in Norway. Psychol Med. 1986;16:39-48.

[10] Huntington G. On chorea. J Neuropsychiatry Clin Neurosci. 2003;15(1):110-2.

[11] Carroll JB, Bates GP, Steffan J, Saft C, Tabrizi SJ. Treating the whole body in Huntington's disease. Lancet Neurol. 2015;14(11):1135-42.

[12] Heemskerk A, Roos RA. Aspiration pneumonia and death in Huntington's disease. PLoS Currents Huntington Disease. 2012. Edition 1. doi: 10.1371/currents.RRN1293 Statistics Norway (2003).

[13] van Walsem MR, Howe EI, Iversen K, Frich JC, Andelic N. Unmet needs for health care and social support services in patients with Huntington's disease: A cross-sectional population-based study. Orphanet J Rare Dis. 2015;10:124.

[14] Chaganti SS, McCusker EA, Loy CT. What do we know about Late Onset Huntington's Disease? J Huntingtons Dis. 2017;6(2):95-103.

[15] Hille ET, Siesling S, Vegter-van der Vlis M, Vandenbroucke JP, Roos RA, Rosendaal FR. Two centuries of mortality in ten large families with Huntington disease: A rising impact of gene carriership. Epidemiology. 1999;10(6): 706-10.

[16] Fiedorowicz JG, Mills JA, Ruggle A, Langbehn D, Paulsen JS; PREDICT-HD Investigators of the Huntington Study Group. Suicidal behavior in prodromal Huntington disease. Neurodegener Dis. 2011;8(6):483-90.

[17] Paulsen JS, Long JD. Onset of Huntington's disease: Can it be purely cognitive? Mov Disord. 2014;29(11):1342-50.

[18] Dale M, Maltby J, Shimozaki S, Cramp R, Rickards H; REGISTRY Investigators of the European Huntington's 
Disease Network. Disease stage, but not sex, predicts depression and psychological distress in Huntington's disease: A European population study. J Psychosom Res. 2016;80:17-22.

[19] Pechlivanis I, Andrich J, Scholz M, Harders A, Saft C, Schmieder K. Chronic subdural haematoma in patients with Huntington's disease. Br J Neurosurg. 2006;20(5):327-9.
[20] Statistics Norway (2003). Population size in Norway 1900-2017. http://www.ssb.no/300132/folkemengde-per-1. januar-fodte-dode-flyttinger-og-folketilvekst-sa-48 